\title{
Entropy Production in Nonequilibrium Systems Described by a Fokker-Planck Equation
}

\author{
Tânia Tomé \\ Instituto de Física \\ Universidade de São Paulo \\ Caixa postal 66318 \\ 05315-970 São Paulo- SP, Brazil
}

Received on 24 September, 2006

\begin{abstract}
We study the entropy production in nonequilibrium systems described by a Fokker-Planck equation. We have devised an expression for the entropy flux in the stationary state. We have found that the entropy flux can be written as an ensemble average of an expression containing the force and its derivative. This result is similar to the one used by Lebowitz and Spohn for system following a Markovian process in discrete space. We have also been able to obtain a fluctuation-dissipation type relation between the dissipated power, which was written as an ensemble average, and the production of entropy for the case of systems in contact with one heat bath. We have applied the results for a simple model for particles subjected to dissipative forces.
\end{abstract}

Keywords: Entropy production; Irreversible systems; Fokker-Planck equation

\section{INTRODUCTION}

In the stationary state, irreversible systems are in a continuous process of production of entropy. A measure of the distance from thermodynamic equilibrium can therefore be given by the production of entropy since this quantity vanishes in equilibrium. The rate of change of the entropy $S$ of a system can be properly decomposed into two contributions [1]

$$
\frac{d S}{d t}=\Pi-\Phi
$$

where $\Pi$ is the entropy production due to irreversible processes ocurring inside the system and $\Phi$ is the entropy flux from the system to the environment. The quantity $\Pi$ is positive definite whereas $\Phi$ can have either sign. In the stationary state the rate of change of the entropy vanishes so that $\Phi=\Pi$. The quantity $\Phi$ is defined as the flux from inside to outside the system, so that it will be positive in the nonequilibrium stationary state.

Although in equilibrium the entropy is a well defined quantity, in nonequilibrium systems the entropy as well as the production of entropy do not have a universal definition. According to Gallavotti the problem of defining entropy in a system out of equilibrium has not been solved yet [2]. In this sense it is interesting to see how one can define those quantities in systems that evolve in time according to specified dynamics. In deterministic Hamiltonian dynamics it is well known that the Gibbs entropy is invariant [3]. This property is a consequence of the incompressibility of the "fluid" that represents the system in phase space. Irreversible systems, on the other hand, are supposed to be described by non-Hamiltonian dynamics, that is, by dynamics coming from nonconservative forces. In this case, the "fluid" in phase space becomes compressible and the production of entropy may be related to the contraction of the phase space $[2,4,5]$.

Here we are concerned with the definition and calculation of the entropy production in nonequilibrium systems described by a Fokker-Planck equation or in an equivalent description by a Langevin equation $[6,7]$. Systems described by a Fokker-Planck equation are systems that follow a Makovian process in continuous time and continuous configuration space. The irreversible character is determined by the type of force entering the Langevin and its associate Fokker-Planck equation. As we shall see, if the forces are nonconservative the resulting entropy production is nonzero and positive. When the force becomes conservative the production of entropy vanishes. The study of production of entropy in systems described by a Markovian process in continuous time but discrete configuration space $[7,8]$ or, in other words, described by a master equation, has been the object of several studies [9-13] including the production of entropy in the majority vote model $[13,14]$. In this study we will relate the entropy production $\Pi$ with the dissipated power $\mathcal{P}$ occurring in nonequilibrium systems subject to nonconservative forces and in contact with a heat bath at a temperature $T$. We will show that in this case $\Pi=\mathscr{P} / T$, which is a fluctuation-dissipation type relation.

\section{FOKKER-PLANCK EQUATION}

Let us consider a set of $n$ interacting particles that evolve in time according to the following coupled set of Langevin equations

$$
\frac{d x_{i}}{d t}=f_{i}(x)+\zeta_{i}(t)
$$

where $x_{i}$ is the position of the $i$-th particle and $x$ stands for the collection of variables $\left\{x_{i}\right\}$. The quantity $f_{i}(x)=f_{i}\left(\left\{x_{i}\right\}\right)$ is the force acting on the $i$-th particle and $\zeta_{i}(t)$ is the additive white noise, that is, a stochastic variable having the properties

$$
\left\langle\zeta_{i}(t)\right\rangle=0,
$$

and

$$
\left\langle\zeta_{i}(t) \zeta_{j}\left(t^{\prime}\right)\right\rangle=2 D_{i} \delta_{i j} \delta\left(t-t^{\prime}\right),
$$

where, for simplicity, we are assuming that $D_{i} \geq 0$ are constants that might be distinct for each particle. 
The associated Fokker-Planck equation, that gives the time evolution of the probability distribution $P(x, t)$, is given by

$$
\frac{\partial}{\partial t} P(x, t)=-\sum_{i} \frac{\partial}{\partial x_{i}}\left[f_{i}(x) P(x, t)\right]+\sum_{i} D_{i} \frac{\partial^{2}}{\partial x_{i}^{2}} P(x, t) .
$$

It is convenient to write down the Fokker-Planck equation as the following continuity equation

$$
\frac{\partial}{\partial t} P(x, t)=-\sum_{i} \frac{\partial}{\partial x_{i}} J_{i}(x, t),
$$

where $J_{i}$ is the $i$-th component of the current of probability $J=\left\{J_{i}\right\}$, defined by

$$
J_{i}(x, t)=f_{i}(x) P(x, t)-D_{i} \frac{\partial}{\partial x_{i}} P(x, t) .
$$

The Fokker-Planck equation has to be solved inside a given region $\mathcal{R}$ of the space spanned by the set of variables $x_{i}$ subject to a prescribed boundary condition which concerns the behavior of $P(x, t)$ and $J_{i}(x, t)$ at the surface $S$ that delimits the region $R$. We will consider two types of boundary conditions: periodic and reflecting. For periodic boundary conditions both quantities are periodic. In this case, the forces $f_{i}(x)$ must be periodic. For the reflecting boundary conditions the component of the current of probability $J$ perpendicular to $S$ vanishes. In this case the component of the force perpendicular to $S$ is assumed to vanish. All results obtained below will be valid under these two types of boundary conditions.

\section{REVERSIBILITY}

We will be concerned here with systems that are far from equilibrium, that is systems that are irreversible even in the stationary state. This means that the current of probability does not vanish in the stationary state. If the current of probability vanishes in the steady state, the system is found to be in a state of thermodynamic equilibrium and we say that the system is reversible. In this case, the entropy production $\Pi$ vanishes and the flux of entropy $\Phi$ vanishes as well. The property of reversibility holds when the forces $f_{i}$ are conservative, that is, when

$$
\frac{\partial f_{i}}{\partial x_{j}}=\frac{\partial f_{j}}{\partial x_{i}}
$$

for any pair $i, j$ and

$$
D_{i}=D
$$

is the same for any particle. In this case the $f_{i}$ is the gradient of a certain potential $V(x)$, that is,

$$
f_{i}(x)=-\frac{\partial V(x)}{\partial x_{i}}
$$

and the stationary probability distribution $P_{e}(x)$ is the solution of

$$
-\frac{\partial V(x)}{\partial x_{i}} P_{e}(x)-D \frac{\partial}{\partial x_{i}} P_{e}(x)=0
$$

that is,

$$
P_{e}(x)=\frac{1}{Z} e^{-V(x) / D}
$$

where $Z$ is a normalization constant. Assuming that $D$ is proportional do the temperature $T$, this is the canonical distribution.

The Langevin equation and its associate Fokker-Planck equations are capable of describing system in thermodynamic equilibrium if $f_{i}$ and $D_{i}$ satisfy the relations (8) and (9). In this case we interpret the equations as the ones appropriate do describe a system in contact with a heat reservoir at temperarature $T$. In the following we will be concerned with systems for which the conditions given by Eqs. (8) and (9) do not hold. The situation in which condition (8) holds but the $D_{i}$ are not the same is appropriate do describe, for instante, the contact of the system with two or more heat reservoirs with distinct temperatures. In this case the stationary state will be a nonequilibrium steady state. Another irreversible situation is found when $D_{i}=D$, but there exists at least one pair $i, j$ for which

$$
\frac{\partial f_{i}}{\partial x_{j}} \neq \frac{\partial f_{j}}{\partial x_{i}}
$$

This describes, for instance, the contact of an irreversible system, containing nonconservative forces, with a heat reservoir. In these nonequilibirum cases the production of entropy $\Pi$ and the flux of entropy $\Phi$ are both nonzero.

\section{ENTROPY}

The Gibbs entropy $S(t)$ of the system at time $t$ is defined by

$$
S(t)=-\int P(x, t) \ln P(x, t) d x,
$$

where $d x=d x_{1} d x_{2} \ldots d x_{n}$. Using the Fokker-Planck equation in the form of Eq. (6), its time derivative can be written as

$$
\frac{d}{d t} S(t)=\int[\ln P(x, t)+1] \sum_{i} \frac{\partial}{\partial x_{i}} J_{i}(x, t) d x .
$$

Integrating by parts we get

$$
\frac{d}{d t} S(t)=-\int \sum_{i} J_{i}(x, t) \frac{\partial}{\partial x_{i}} \ln P(x, t) d x
$$

Now from the definition of current of probability given by Eq. (7) it follows that

$$
D_{i} \frac{\partial}{\partial x_{i}} \ln P(x, t)=f_{i}(x)-\frac{J_{i}(x, t)}{P(x, t)} .
$$

Therefore, we may write

$$
\frac{d}{d t} S(t)=
$$




$$
=-\int \sum_{i} \frac{1}{D_{i}} J_{i}(x, t) f_{i}(x) d x+\int \sum_{i} \frac{\left[J_{i}(x, t)\right]^{2}}{D_{i} P(x, t)} d x .
$$

The last term is clearly positive definite. We identify it with the entropy production, that is,

$$
\Pi=\int \sum_{i} \frac{\left[J_{i}(x, t)\right]^{2}}{D_{i} P(x, t)} d x .
$$

Comparing with Eq. (1), it follows that the entropy flux $\Phi$ should be given by

$$
\Phi=\int \sum_{i} \frac{1}{D_{i}} J_{i}(x, t) f_{i}(x) d x .
$$

Multiplying the density of current given by Eq. (7) by $f_{i}$ and integrating we have

$$
\begin{gathered}
\int J_{i}(x, t) f_{i}(x) d x= \\
=\int\left[f_{i}(x)\right]^{2} P(x, t) d x-D_{i} \int f_{i}(x) \frac{\partial}{\partial x_{i}} P(x, t) d x .
\end{gathered}
$$

Integrating the last term by parts, we get the following expression for the entropy flux,

$$
\Phi=\int \sum_{i}\left\{\frac{1}{D_{i}}\left[f_{i}(x)\right]^{2}+f_{i i}(x)\right\} P(x, t) d x,
$$

where $f_{i i}(x)=\partial f_{i}(x) / \partial x_{i}$. The right-hand side can be written as an average over the probability distribution $P(x, t)$, that is,

$$
\Phi=\left\langle\sum_{i}\left\{\frac{1}{D_{i}}\left[f_{i}(x)\right]^{2}+f_{i i}(x)\right\}\right\rangle .
$$

\section{MASTER EQUATION}

In this section we show that a discretized Fokker-Planck equation can be viewed as a master equation. To this end let us consider a Fokker-Planck equation in one variable

$$
\frac{\partial}{\partial t} P(x, t)=-\frac{\partial}{\partial x}\left[f(x) P(x, t]+D \frac{\partial^{2}}{\partial x^{2}} P(x, t) .\right.
$$

If we use the discretization $x=n a$ then this equation can be written as

$$
\begin{gathered}
\frac{d}{d t} P_{n}(t)=-\frac{1}{a}\left[f_{n+1} P_{n+1}(t)-f_{n} P_{n}(t)\right]+ \\
+\frac{D}{a^{2}}\left[P_{n+1}(t)-2 P_{n}(t)+P_{n-1}(t)\right],
\end{gathered}
$$

where $P_{n}(t)=P(x, t)$ and $f_{n}=f(x)$, which in turn can be identified as the master equation

$$
\frac{d}{d t} P_{n}(t)=w_{n-1}^{+} P_{n-1}(t)-w_{n}^{+} P_{n}(t)+
$$

$$
+w_{n+1}^{-} P_{n+1}(t)-w_{n}^{-} P_{n}(t)
$$

where

$$
w_{n}^{+}=\frac{D}{a^{2}}
$$

is the transition probability of jumping from $n$ to $n+1$ and

$$
w_{n}^{-}=\frac{D}{a^{2}}-\frac{f_{n}}{a}
$$

is the transition probability of jumping from $n$ to $n-1$.

According to Lebowitz and Spohn [10] the flux of entropy in a system governed by a master equation is given by

$$
\Phi=\left\langle w_{n}^{+} \ln \frac{w_{n}^{+}}{w_{n+1}^{-}}+w_{n}^{-} \ln \frac{w_{n}^{-}}{w_{n-1}^{+}}\right\rangle,
$$

Now, if we take the limit $a \rightarrow 0$ it is straightforward to show that this expression becomes

$$
\Phi=\left\langle\frac{1}{D}[f(x)]^{2}+f^{\prime}(x)\right\rangle,
$$

which is the expression given by Eq. (23) for the particular case of one variable.

\section{TIME AVERAGE OF A STATE FUNCTION}

Let us consider the time average of a state function $E(x)$ given by

$$
U(t)=\int E(x) P(x, t) d x .
$$

We have

$$
\frac{d}{d t} U(t)=-\int E(x) \sum_{i} \frac{\partial}{\partial x_{i}} J_{i}(x, t) d x .
$$

Integrating by parts,

$$
\frac{d}{d t} U(t)=\int \sum_{i} J_{i}(x, t) E_{i}(x) d x,
$$

where $E_{i}(x)=\partial E(x) / \partial x_{i}$, or

$$
\begin{gathered}
\frac{d}{d t} U(t)=\int \sum_{i} E_{i}(x) f_{i}(x) P(x, t) d x- \\
-\int \sum_{i} D_{i} E_{i}(x) \frac{\partial}{\partial x_{i}} P(x, t) d x .
\end{gathered}
$$

Integrating the last term by parts we get

$$
\begin{gathered}
\frac{d}{d t} U(t)=\int \sum_{i} E_{i}(x) f_{i}(x) P(x, t) d x+ \\
+\int \sum_{i} D_{i} E_{i i}(x) P(x, t) d x,
\end{gathered}
$$


where $E_{i i}(x)=\partial E_{i}(x) / \partial x_{i}$ and we have assumed that $E_{i}(x)$ is either periodic or vanishes at the boundary, depending on the type of boundary condition. The right-hand side can therefore be written as an average,

$$
\frac{d}{d t} U(t)=\left\langle\sum_{i}\left\{E_{i}(x) f_{i}(x)+D_{i} E_{i i}(x)\right\}\right\rangle .
$$

\section{DISSIPATED POWER}

The dissipated energy per unit time due to the forces $f_{i}$, or the dissipated power of the forces $f_{i}$, is determined by

$$
\mathcal{P}=\left\langle\sum_{i} f_{i} \frac{d x_{i}}{d t}\right\rangle .
$$

If we use the Langevin equation this can be written as

$$
\mathcal{P}=\left\langle\sum_{i}\left[f_{i}\right]^{2}\right\rangle+\left\langle\sum_{i} f_{i} \zeta_{i}(t)\right\rangle
$$

Using now the Stratonovich prescription, it is possible to show that $\left\langle f_{i} \zeta_{i}(t)\right\rangle=D_{i}\left\langle f_{i i}\right\rangle$, where $\left.f_{i i}=\partial f_{i} / \partial x_{i}\right\rangle$, so that

$$
\mathcal{P}=\left\langle\sum_{i}\left\{\left[f_{i}\right]^{2}+D_{i} f_{i i}\right\}\right\rangle .
$$

This expression has been obtained before [15] by means of a similar reasoning. Using a reasoning similar to those used before we can write this expression as

$$
\mathcal{P}=\int \sum_{i} J_{i}(x, t) f_{i}(x) .
$$

It is interesting to notice that there is a simple relationship between the dissipated power and the entropy flux when the system is in contact with a heat reservoir. In this case, as we have seen, $D_{i}=D$ where $D$ is proportional do the temperature of the reservoir. Comparing these two expression with those for the entropy flux $\Phi$ we see that

$$
\Phi=\frac{P}{D} .
$$

Writing $D=k T$ and defining $\Phi^{*}=k \Phi$ we see that $\Phi^{*}=\mathcal{P} / T$. We remark that this relation is valid for systems that are in contact with a heat reservoir but subject to nonconservative forces.

\section{NONCONSERVATIVE FORCE}

From now one we will consider the case such that $D_{i}=D$, which correponds to the description of a system in contact with a heat reservoir. In the stationary state, expression (23) for the entropy flux $\Phi$ gives also the production of entropy $\Pi$ since the variation in entropy $d S / d t$ vanishes. However, if the system is in a transient state this expression will be a sum of two terms: one is the production of entropy $\Pi$ and the other is minus the entropy variation $-d S / d t$ of the system. Let us compute each of these terms. To this end we start by splitting the force $f_{i}(x)$ into two parts

$$
f_{i}(x)=f_{i}^{C}(x)+f_{i}^{D}(x)
$$

such that $f_{i}^{C}(x)$ is conservative, that is, $f_{i}^{C}(x)$ is the gradient of a potencial $E(x)$, or

$$
f_{i}^{C}=-\frac{\partial E}{\partial x_{i}},
$$

and $f_{i}^{D}(x)$ is a nonconservative force with a vanishing divergence (solenoidal force), that is,

$$
\sum_{i} \frac{\partial f_{i}^{D}}{\partial x_{i}}=0 .
$$

That such a splitting can always be done can be seen as follows. This last equation implies that

$$
\sum_{i} \frac{\partial f_{i}}{\partial x_{i}}=\sum_{i} \frac{\partial f_{i}^{C}}{\partial x_{i}}
$$

or yet

$$
\sum_{i} \frac{\partial f_{i}}{\partial x_{i}}=-\sum_{i} \frac{\partial^{2} E}{\partial x_{i}^{2}}
$$

Therefore, if we are given a generic force $f_{i}(x)$, this last equation can be solved to obtain $E(x)$. From $E(x)$ we get $f_{i}^{C}(x)$ by means of Eq. (43) and $f_{i}^{D}(x)$ by using Eq. (42).

The flux of entropy is written as a sum of two terms, $\Phi=$ $\Phi_{C}+\Phi_{D}$, where

$$
\Phi_{C}=\left\langle\sum_{i}\left\{\frac{1}{D} f_{i}^{C}(x) f_{i}(x)+f_{i i}^{C}(x)\right\}\right\rangle .
$$

and

$$
\Phi_{D}=\frac{1}{D}\left\langle\sum_{i} f_{i}^{D}(x) f_{i}(x)\right\rangle .
$$

Now, since $f_{i}^{C}(x)$ is the gradient of a state function $E(x)$ we may use the identification $f_{i}^{C}=E_{i}$ and Eq. (36) to conclude that

$$
\Phi_{C}=\frac{1}{D} \frac{d}{d t}\langle E(x)\rangle
$$

In the stationary state, the right-hand side vanishes so that $\Phi_{C}$ vanishes and $\Pi=\Phi=\Phi_{D}$.

\section{A SIMPLE MODEL}

Let us consider a simple model with two variables, for which the force is given by

$$
\mathbf{f}=-K \mathbf{r}+A \hat{z} \times \mathbf{r}
$$


where $K$ and $A$ are two parameters, and $\mathbf{r}=x_{1} \hat{x}+x_{2} \hat{y}$ and $\mathbf{f}=f_{1} \hat{x}+f_{2} \hat{y}$. As long as $A \neq 0$ the force is nonconservative since $\nabla \times \mathbf{f}=-2 A \hat{z} \neq 0$. In this case

$$
\Phi_{C}=\frac{1}{D}\left\{K^{2}\left\langle\mathbf{r}^{2}\right\rangle-2 D K\right\}
$$

and

$$
\Phi_{D}=\frac{1}{D} A^{2}\left\langle\mathbf{r}^{2}\right\rangle
$$

The Fokker-Planck equation is

$$
\frac{\partial P}{\partial t}=-\nabla \cdot(\mathbf{f} P)+D \nabla^{2} P
$$

which can be written as

$$
\frac{\partial P}{\partial t}=K \nabla \cdot(\mathbf{r} P)-A \hat{z} \cdot \mathbf{r} \times \nabla P+D \nabla^{2} P .
$$

A solution of this equation can be obtained by assuming that $P$ is a function of $|\mathbf{r}|=r$ only. The vector product term in (54) then vanishes and the equation becomes a Fokker-Planck equation for a conservative force given by $\mathbf{f}=-K \mathbf{r}$. The stationary solution is then

$$
P(\mathbf{r})=\frac{K}{2 \pi D} e^{-K r^{2} / 2 D}
$$

from which we get $\left\langle r^{2}\right\rangle=2 D / K$, and the results $\Phi_{C}=0$ and

$$
\Pi=\Phi_{D}=\frac{2 A^{2}}{K} .
$$

The entropy production $\Pi$ is then proportional to $A^{2}$ and vanishes when $A \rightarrow 0$, that is, in the equilibrium case.

We may also determine the stationary current of probability. From its definition

$$
\mathbf{J}(\mathbf{r}) \mathbf{J}(\mathbf{r})=\mathbf{f}(\mathbf{r}) P(\mathbf{r})-D \nabla P(\mathbf{r})
$$

we obtain the result

$$
\mathbf{J}(\mathbf{r})=A(\hat{z} \times \mathbf{r}) P(\mathbf{r}) .
$$

Hence, the current of probability is proportional to $A$ and also vaninhes in the equilibrium state, as expected.

\section{CONCLUSION}

We have devised an expression for the entropy flux in the stationary state for systems that follow a Fokker-Planck equation. We have found that the entropy flux can be written as an average value of an expression containing the force and its derivative. This result can be compared with the one by Lebowitz and Spohn. In fact they were able to show that the entropy flux for markovian processes can be calculated as an average of an state function at the steady state. We have also shown that for a simple model for particles subjected to dissipative forces the entropy flux, which equals the entropy production, in the steady state is nonzero.
[1] G. Nicolis and I. Prigogine, Self-Organization in Nonequilibrium Systems (Wiley, New York, 1977).

[2] G. Gallavotti, Chaos 14, 680 (2004).

[3] J. W. Gibbs, Elementary Principles in Statistical Mechanics, Yale University Press, New Haven, 1902.

[4] G. Gallavotti and E. G. D. Cohen, Phys. Rev. E 69, 035104 (2004).

[5] D. J. Evans, D. J. Searles, and L. Rondoni, Phys. Rev. E 71 056120 (2005)

[6] N. G. van Kampen, Stochastic Processes in Physics and Chemistry, North-Holland Amsterdam, 1981.

[7] T. Tomé e M. J. de Oliveira, Dinâmica Estocástica e Irreversibilidade, Editora da Universidade de São Paulo, São Paulo,
2001.

[8] T. M. Liggett, Interacting Particle Systems, Springer-Verlag, New York, 1985.

[9] C. Maes, J. Stat. Phys. 95, 367 (1999).

[10] J. L. Lebowitz and H. Spohn, J. Stat. Phys. 95, 333 (1999).

[11] C. Maes, F. Redig, and A. Van Moffaert, J. Math. Phys. 41, $1528(2000)$.

[12] C. Maes and K. Netočný J. Stat. Phys. 11, 269 (2003).

[13] L. Crochik and T.Tomé, Phys. Rev. E 72, 026130 (2005).

[14] M. J. de Oliveira, J. Stat. Phys. 66, 273 (1992).

[15] T. Tomé and M. J. de Oliveira, Braz. J. Phys. 27, 525 (1997). 